Full Paper

\title{
Effect of weight reduction with dietary intervention on arterial distensibility and endothelial function in obese men
}

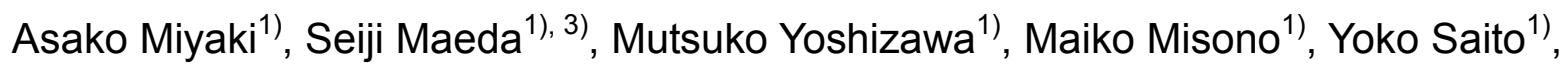 \\ Hiroyuki Sasai ${ }^{1)}$, Takayuki Endo ${ }^{1)}$, Yoshio Nakata $^{2)}$, Kiyoji Tanaka ${ }^{1)}$, and Ryuichi Ajisaka ${ }^{1)}$
}

From the 1) Institute of Health and Sport Sciences, 2) Institute of Clinical Medicine, and 3) Center for Tsukuba Advanced Research Alliance, University of Tsukuba, Tsukuba, Ibaraki, Japan

Running title: Weight reduction and arterial function

Grants: This work was supported by Grants-in-Aid for Scientific Research from the Ministry of Education, Culture, Sports, Science and Technology of Japan (18300215, 18650186).

Asako Miyaki: miyakiasako@yahoo.co.jp, Seiji Maeda: smaeda@tara.tsukuba.ac.jp, Mutsuko Yoshizawa: myoshizawa@med.taiiku.tsukuba.ac.jp, Maiko Misono:

maikomisono@med.taiiku.tsukuba.ac.jp, Yoko Saito: yoko_s@med.taiiku.tsukuba.ac.jp, Hiroyuki Sasai: sasai@stat.taiiku.tsukuba.ac.jp, Takayuki Endo: tendo@stat.taiiku.tsukuba.ac.jp, Yoshio Nakata: nakata@md.tsukuba.ac.jp, Kiyoji Tanaka: tanaka@taiiku.tsukuba.ac.jp, Ryuichi Ajisaka: ajisakas@taiiku.tsukuba.ac.jp

Address for Correspondence:

Seiji Maeda, Ph.D.

Center for Tsukuba Advanced Research Alliance

University of Tsukuba

Tsukuba, Ibaraki 305-8577

Japan

(TEL) +81 29-853-2683

(FAX) +81 29-853-2986

E-mail: smaeda@tara.tsukuba.ac.jp

(20 pages [2 Table and 4 Figures]) 
Weight reduction and arterial function. Miyaki et al. Page 1

\section{ABSTRACT}

Obesity is an independent risk factor for cardiovascular morbidity and mortality.

Reduction in central arterial distensibility has recently been identified as a powerful and independent risk factor for cardiovascular disease. However, the effect of weight reduction on central arterial distensibility and endothelial function in obese subjects has not yet been clarified. We investigated whether low-calorie diet-induced weight reduction affects central arterial distensibility and endothelial function in middle-aged obese men. Twelve obese men (age: $45 \pm 2$ yrs, body mass index: $30 \pm 1 \mathrm{~kg} / \mathrm{m}^{2}$ ) completed a 12-week dietary intervention. Caloric restriction significantly reduced body weight and resulted in a significant decrease in body mass index. After the weight reduction program, carotid arterial compliance (via simultaneous B-mode ultrasound and arterial applanation tonometry on the common carotid artery) significantly increased and $\beta$-stiffness index, an index of arterial compliance adjusted for distending pressure, significantly decreased. Additionally, aortic pulse-wave velocity, an index of arterial stiffness, significantly decreased after weight reduction. Concentrations of plasma endothelin-1 (ET-1), a potent vasoconstrictor peptide produced by vascular endothelial cells, significantly decreased and plasma nitric oxide (NO) [measured as the stable end product (nitrite/nitrate)], a potent vasodilator produced by vascular endothelial cells, significantly increased after the program. Weight reduction by low-calorie diet in obese men increases central arterial distensibility. This increase in central arterial distensibility may contribute to the improvement in endothelial function, as noted by a decrease in ET-1 and an increase in NO, after weight reduction.

Keywords: diet, arterial compliance, endothelial function 
Weight reduction and arterial function. Miyaki et al. Page 2

\section{INTRODUCTION}

The vasculature was once considered to be a simple passive conduit.

However, it is now recognized to be a complex organ capable of producing a number of local vasoactive factors, actively buffering cardiac pulsation. Reduction in central arterial distensibility is believed to contribute to the pathophysiology of cardiovascular disease and has recently been identified as a powerful and independent risk factor for cardiovascular disease $(1,2)$.

The prevalence of obesity has been increasing worldwide. Obesity has been identified as an independent risk factor for cardiovascular morbidity and mortality $(3,4)$. Several studies have investigated the relationship between obesity and large arterial function with contradictory results. In some studies, obese subjects have been found to have lower degrees of arterial distensibility $(5,6)$, while in other studies they had higher degrees of distensibility $(7,8)$. Thus, the relationship between obesity and arterial distensibility has not yet been clarified. On the other hand, endothelial dysfunction is a common abnormality in obesity $(9,10)$. Vascular endothelial cells play an important role in the regulation of vascular activity by producing vasoactive substances, such as endothelin-1 (ET-1) and nitric oxide (NO). ET-1 is a potent vasoconstrictor peptide produced by vascular endothelial cells (11). NO produced by vascular endothelial cells has a potent vasodilator effect (12). Therefore, altered ET-1 and NO may reflect changes in endothelial function and could have important clinical significance.

The purpose of the present study was to examine whether low-calorie diet-induced weight reduction affects central arterial distensibility in middle-aged obese men, and if so, whether the endothelial function, as assessed by ET-1 and NO concentrations, participates in the mechanism underlying the adaptation of central arterial distensibility to weight loss. We hypothesized that weight loss can increase 
Weight reduction and arterial function. Miyaki et al. Page 3

central arterial distensibility in obese men, and ET-1 and NO participate in the mechanism underlying this adaptation of central arterial distensibility to weight loss.

To test our hypothesis, we measured carotid arterial compliance; $\beta$-stiffness index, an index of arterial compliance adjusted for distending pressure; aortic pulse-wave velocity (PWV), a traditional index of arterial stiffness; and plasma ET-1 and NO concentrations before and after a 12-week dietary intervention in obese men.

\section{METHODS}

Subjects. Twelve obese men participated in the study (45 $\pm 2 \mathrm{yrs}$, body mass index [BMI]: $30 \pm 1 \mathrm{~kg} / \mathrm{m}^{2}$ ). Candidates who were current smokers or who were taking any medications were excluded. All subjects had no apparent cardiovascular disease as assessed by medical history and physical examination. This study was reviewed and approved by the Institutional Review Board at the University of Tsukuba. All potential risks and procedures of the study were explained to the subjects, and they gave written informed consent to participate in the study.

Experimental Design. All obese men were studied before and after 12 weeks of a diet-induced weight reduction program (i.e., an energy-restricted diet program). All measurements were performed after abstinence from caffeine and an overnight fast. Subjects were studied under supine resting conditions in a quiet, temperature-controlled room $\left(24-26^{\circ} \mathrm{C}\right)$. All measurements were performed after a resting period of at least $20 \mathrm{~min}$.

Dietary Intervention. The protocol used has been reported as a low-calorie diet intervention with effects on body composition, body fat distribution, and coronary heart disease risk factors (13). Based on the diet protocol in this study, all subjects were instructed to take meals consisting on average of $420 \mathrm{kcal}$ of protein, $840 \mathrm{kcal}$ of 
Weight reduction and arterial function. Miyaki et al. Page 4

carbohydrate, and $420 \mathrm{kcal}$ of fat per day (total: 1,680 kcal/day). Subjects kept daily food diaries during the 12-week intervention period and learned about proper daily nutrition (well-balanced protein, carbohydrate, fat, amino acids, vitamins, and minerals) through weekly lectures and counseling by skilled dieticians.

Abdominal Adipose Tissue Area by CT. Visceral fat area and subcutaneous fat area $\left(\mathrm{cm}^{2}\right)$ were measured at the level of the umbilicus (L4-L5) using CT (computed tomography) scans (Somatom AR.C, Siemens, Erlangen, Germany) performed on subjects in the supine position. Visceral fat area and subcutaneous fat area were calculated using a computer software program (FatScan, N2system, Osaka, Japan). The intra-class correlation for repeated determinations in the laboratory which performed this measurement was 0.99 .

Blood Pressure Measurement. The supine systolic blood pressure, diastolic blood pressure, mean arterial pressure, and heart rate were recorded from the left arm using a semi-automated device (Dinamap, Johnson \& Johnson, New Jersey, USA).

\section{Carotid Artery Compliance.}

The combination of ultrasound imaging of a common carotid artery with simultaneous applanation of tonometrically obtained arterial pressure from the contralateral carotid artery permits noninvasive determination of dynamic arterial compliance. Subjects were studied under quiet resting conditions while they were in the supine position. Common carotid artery diameter was measured from the images derived from an ultrasound machine (EnVisor, Koninklijke Philips Electronics, Eindhoven, Netherlands) equipped with a high-resolution (7.5 MHz) linear-array transducer. Longitudinal images of the cephalic portion of the common carotid artery were acquired 1 to $2 \mathrm{~cm}$ proximal to the carotid bulb with the transducer placed at a 90 degree angle to the vessel so that near and far wall interfaces were clearly 
Weight reduction and arterial function. Miyaki et al. Page 5

discernible. These images were recorded on a computer recorder for later offline analysis. The computer images were analyzed with the use of image analysis software. All image analyses were performed by the same investigator. Time points that corresponded with maximal systolic expansion of the carotid artery and basal (minimum) diastolic relaxation were selected. The distances (or the diameter) between the vessel far-wall boundary, corresponding to the interface of the adventitia and media, were then measured.

To characterize central arterial compliance as comprehensively as possible, two different measures, arterial compliance (14) and beta-stiffness index (15), were calculated. Cross-sectional compliance (CC) was calculated from the change in cross-sectional area $(d A)$ and local pulse pressure $(d P)$ using the formula $C C=d A / d P$. $d A$ was calculated as $d A=\pi \cdot[(D+d D) / 2]^{2}-\pi \cdot(D / 2)^{2}$. Pressure wave forms of the left common carotid artery were recorded with an applanation tonometry device (formPWVIABI, Colin Medical Technology, Komaki, Japan) and calibrated by equating the carotid mean arterial and diastolic blood pressure to that of the brachial artery (16).

The $\beta$-stiffness index provides an index of arterial compliance adjusted for distending pressure. The $\beta$-stiffness index was calculated using the equation $\beta=\ln$ $(\mathrm{Ps} / \mathrm{Pd}) /[(\mathrm{Ds}-\mathrm{Dd}) / \mathrm{Dd})]$, where Ds and Dd are the maximum and minimum arterial diameters and Ps and Pd are the highest and lowest blood pressures, respectively (15).

Aortic PWV. Aortic PWV was measured using applanation tonometry as previously described (17) with minor modifications. Briefly, carotid and femoral arterial pulse waves were obtained in triplicate using arterial applanation tonometry incorporating an array of 15 transducers (formPWVIABI, Colin Medical Technology, 
Weight reduction and arterial function. Miyaki et al. Page 6

Komaki, Japan). The distance traveled by the pulse waves was assessed in triplicate with a random zero-length measurement over the surface of the body with a nonelastic tape measure. Pulse-wave transit time was determined from the time delay between the proximal and distal "foot" waveforms. The foot of the wave was identified as the commencement of the sharp systolic upstroke, which was automatically detected. Aortic PWV was calculated as the distance divided by the transit time.

Blood Biochemistry. Each blood sample was placed in a chilled tube containing aprotinin (300 kalikrein-inactivating $\mathrm{U} / \mathrm{ml}$ ) and EDTA $(2 \mathrm{mg} / \mathrm{ml})$ and was then centrifuged at $2000 \mathrm{~g}$ for $15 \mathrm{~min}$ at the $4^{\circ} \mathrm{C}$. The plasma was stored at $-80^{\circ} \mathrm{C}$ until assayed. Plasma concentrations of ET-1 were determined using a sandwich-EIA Kit (Immuno-Biological Laboratories, Fujioka, Japan). The ET-1 assay was carried out as previously described (18). Plasma NO (measured as its stable end product, nitrite/nitrate [NOx]) concentration was determined as previously described (19). Serum concentrations of cholesterol and triglycerides and plasma concentrations of glucose were determined using standard enzymatic techniques.

Statistical Analysis. Data are expressed as means \pm SE. To evaluate differences in the levels before and after the weight reduction program in obese men, the Student's $t$-test for paired values was used. $P<0.05$ was accepted as significant.

\section{RESULTS}

Table 1 shows the characteristics of obese men before and after 12 weeks of the weight reduction program. Body weight, BMI, and body fat areas markedly decreased after the weight reduction program (Table 1). Serum concentrations of cholesterol and triglycerides and plasma concentrations of glucose also significantly 
Weight reduction and arterial function. Miyaki et al. Page 7

decreased after the program (Table 1). After the weight reduction program, systolic blood pressure, diastolic blood pressure, mean arterial pressure, pulse pressure, and heart rate significantly decreased (Table 2).

The arterial compliance significantly increased and $\beta$-stiffness index significantly decreased after the weight reduction program (Fig. 1). Additionally, aortic PWV significantly decreased after weight reduction (Fig. 2). Thus, weight reduction by a low-calorie diet in obese men increased central arterial distensibility.

The plasma ET-1 concentration significantly decreased after the weight reduction program (Fig. 3). On the other hand, the plasma concentration of NOx significantly increased with weight loss (Fig. 4).

\section{DISCUSSION}

In the present study, we determined central arterial distensibility and endothelial function in obese men before and after a 12-week diet-induced weight reduction program. After the program due to which body weight and BMI markedly decreased, carotid arterial compliance significantly increased and beta-stiffness index significantly decreased. Additionally, aortic PWV significantly decreased after weight loss. Thus, weight reduction by low-calorie diet in obese men induced an increase in central arterial distensibility. We also demonstrated that the low-calorie diet in obese men significantly decreased plasma ET-1 and significantly increased plasma NOx, a stable end product of NO, suggesting an improvement in endothelial function. Therefore, we suggest that increased central arterial distensibility with weight loss may contribute to the improvement in endothelial function after weight reduction.

Obesity is strongly associated with cardiovascular disease $(3,4,9,10)$. On the other hand, reduced arterial distensibility has been implicated in the 
Weight reduction and arterial function. Miyaki et al. Page 8

pathophysiology of cardiovascular disease and has been identified as an independent risk factor for cardiovascular disease $(1,2)$. The relationship between obesity and large arterial function has been investigated in several studies. The results of these studies vary considerably. In some studies, obese individuals have been found to have a lower degree of arterial distensibility $(5,6)$, while in other studies they have had a higher degree of arterial distensibility $(7,8)$. The arterial distensibility in middle-aged obese humans in the present study was clearly lower than in middle-aged normal weight humans $(15,20)$. These discordant results may be due to the method used to describe arterial distensibility. Few interventional studies have investigated the effects of weight loss on arterial distensibility. Toto-Moukouo et al. (21) have shown that aortic PWV decreased with weight reduction in obese hypertensive subjects. Weight reduction has also been associated with increased arterial compliance in obese men and women $(22,23)$. In the present study, we demonstrated increased arterial compliance, decreased $\beta$-stiffness index, and reduced aortic PWV with weight loss in obese men. These findings strongly suggest that weight reduction in obese men induces increased central arterial distensibility, which may have beneficial effects on the prevention of cardiovascular disease.

Obesity is strongly associated with endothelial dysfunction $[9,10]$ that may play a role in the development of decreased arterial distensibility. Vascular endothelial cells produce ET-1 and NO $(11,12)$. ET-1 produced by vascular endothelial cells has potent vasoconstrictor activity and potent proliferative effects on vascular smooth muscle cells $(11,24)$. Furthermore, arterial distensibility was decreased by intra-arterial infusion of ET-1 and increased by the administration of an ET-1 receptor antagonist $(25,26)$. NO produced by vascular endothelial cells has a potent vasodilator effect and plays an important role in the regulation of 
Weight reduction and arterial function. Miyaki et al. Page 9

platelet-vessel wall interactions and vascular resistance and growth (12). Arterial distensibility decreased following intra-arterial infusion of an NO synthase inhibitor (27). These findings suggest that endogenous ET-1 and NO participate in the regulation of arterial distensibility. In the present study, we showed decreased plasma ET-1 concentrations and increased plasma NOx concentrations with weight reduction in obese men. On the basis of results from past studies along with the present results, we propose that the increase in central arterial distensibility with weight loss may contribute to improved endothelial function, as assessed by decreased ET-1 and increased NO, after weight reduction.

In conclusion, the present study demonstrated that low-calorie diet-induced weight reduction increased central arterial distensibility in middle-aged obese men. The improvements in arterial compliance were associated with a reduction in plasma ET-1 and an increase in plasma NOx, a stable end product of NO, and consequently, improved endothelial function. These results indicate that ET-1 and NO participate in the mechanism underlying the increase in central arterial distensibility with weight loss. 
Weight reduction and arterial function. Miyaki et al. Page 10

\section{ACKNOWLEDGEMENTS}

This work was supported by Grants-in-Aid for Scientific Research from the Ministry of Education, Culture, Sports, Science and Technology of Japan (18300215, 18650186). 
Weight reduction and arterial function. Miyaki et al. Page 11

\section{REFERENCES}

1. Safar ME, London GM. Therapeutic studies and arterial stiffness in hypertension: recommendations of the European Society of Hypertension. The Clinical Committee of Arterial Structure and Function. J Hypertens 2000; 18: 1527-1535.

2. Hodes RJ, Lakatta EG, McNeil CT. Another modifiable risk factor for cardiovascular disease? Some evidence points to arterial stiffness. J Am Geriatr Soc 1995; 43: 581-582.

3. Hubert HB, Manning F. Obesity is an independent risk factor for cardiovascular disease: a 26-year follow up of participants in the Framingham Heart Study. Circulation 1983; 67: 968-977.

4. Zhang C, Rexrode KM, van Dam RM, Li TY, Hu FB. Abdominal obesity and the risk of all-cause, cardiovascular, and cancer mortality. Circulation 2008; 117 : 1658-1667.

5. Danias PG, Tritos NA, Stuber M, Botnar RM, Kissinger KV, Manning WJ. Comparison of aortic elasticity determined by cardiovascular magnetic resonance imaging in obese versus lean adults. Am J Cardiol 2003; 91: 195-199.

6. Sutton-Tyrrell K, Newman A, Simonsick EM et al. Aortic stiffness is associated with visceral adiposity in older adults enrolled in the Study of Health, Aging, and Body Composition. Hypertension 2001; 38: 429-433.

7. Oren S, Grossman E, Frohlich ED. Arterial and venous compliance in obese and nonobese subjects. Am J Cardiol 1996; 77: 665-667.

8. Mangoni AA, Giannattasio C, Brunani A et al. Radial artery compliance in young, obese, normotensive subjects. Hypertension 1995; 26: 984-988.

9. Van Guilder GP, Stauffer BL, Greiner JJ, DeSouza CA. Impaired endothelium-dependent vasodilation in overweight and obese adults humans is not limited to muscarinic receptor agonists. Am J Physiol. 2008; 294: H1685-92.

10. Brook RD, Bard RL, Rubenfire M, Ridker PM, Rajagopalan S. Useflness of visceral obesity(Waist/Hip ratio) in predicting vascular endothelial function in healthy overweight adults. Am J Cardiol. 2001; 88: 1264-1269. 
Weight reduction and arterial function. Miyaki et al. Page 12

11. Yanagisawa $\mathrm{M}$, Kurihara $\mathrm{H}$, Kimura $\mathrm{S}$ et al. A novel potent vasoconstrictor peptide produced by vascular endothelial cells. Nature 1988; 332: 411-415.

12. Moncada S, Palmer RM, Higgs EA. Nitric oxide: physiology, pathophysiology, and pharmacology. Pharmacol Rev 1991; 43: 102-142.

13. Okura T, Nakata Y, Yamabuki K, Tanaka K. Regional body composition changes exhibit opposing effects on coronary heart disease risk factors. Arterioscler Thromb Vasc Biol 2004; 24: 923-929.

14. Van Bortel LM, Duprez D, Starmans-Kool MJ et al. Clinical applications of arterial stiffness, task force 3: Recommendations for user producers. Am J Hypertens 2002; 15: 445-452.

15. Hirai T, Sasayama S, Kawasaki T, Yagi S. Stiffness of systemic arteries in patients with myocardial infarction: a noninvasive method to predict severity of coronary atherosclerosis. Circulation 1989; 80: 78-86.

16. Nichols WW, O'Rourke M. Sphygmocardiography, in Nichols WW, O'Rourke M (eds): MacDnald's blood flow in arteries. Theoretical, experimental and clinical principles ( $4^{\text {th }}$ edition). Arnold E: London-Sydney-Auckland; 1998; pp453-476.

17. Cortez-Cooper MY, Supak JA, Tanaka H. A new device for automatic measurements of arterial stiffness and ankle-brachial index. Am J Cardiol 2003; 91: 1519-1522.

18. Maeda S, Miyauchi T, Sakane M et al. Does endothelin-1 participate in the exercise -induced changes of blood flow distribution of muscles in humans? J Appl Physiol 1997; 82: 1107 -1111.

19. Maeda S, Tanabe T, Otsuki T et al. Moderate regular exercise increases basal production of nitric oxide in elderly women. Hypertens Res 2004; 27: 947-953.

20. Hayashi K, Sugawara J, Komine H, Maeda S, Yokoi T. Effects of aerobic exercise training on stiffness of central and peripheral arteries in middle-aged sedentary men. Jpn J Physiol 2005; 55: 235-239

21. Toto-Moukouo JJ, Achimastos A, Asmar RG, Hugues CJ, Safar ME. Pulse wave 
Weight reduction and arterial function. Miyaki et al. Page 13

velocity in patients with obesity and hypertension. Am Heart J 1986; 112: 136-140.

22. Yamashita T, Sasahara T, Pomeroy SE, Collier G, Nestel PJ. Arterial compliance, blood pressure, plasma leptin, and plasma lipids in women are improved with weight reduction equally with a meatbased diet and a plant-based diet. Metabolism 1998; 47: 1308-1314.

23. Balkestein EJ, Van Aggel-Leijssen DP, Van Baak MA, Struijker-Boudier HA, Van Bortel LM. The effect of weight loss with or without exercise training on large artery compliance in healthy obese men. J Hypertens 1999; 17: 1831-1835.

24. Rubanyi GM, Polokoff MA. Endothelin; molecular biology, biochemistry, pharmacology, physiology, and pathophysiology.

Pharmacol Rev 1994; 46: 325-415.

25. McEniery CM, Qasem A, Schmitt M, Avolio AP, Cockcroft JR, Wilkinson IB. Endothelin-1 regulates arterial pulse wave velocity in vivo. J Am Coll Cardiol 2003; 42: 1975-1981.

26. Vuurmans TJ, Boer P, Koomans HA. Effects of endothelin-1 and endothelin-1 receptor blockade on cardiac output, aortic pressure, and pulse wave velocity in humans. Hypertension 2003; 41: 1253-1258.

27. Wilkinson IB, Qasem A, McEniery CM, Webb DJ, Avolio AP, Cockcroft JR. Nitric oxide regulates local arterial distensibility in vivo.

Circulation 2002; 105: 213-217. 
Weight reduction and arterial function. Miyaki et al. Page 14

\section{FIGURE LEGENDS}

Figure 1. Arterial compliance (A) and $\beta$-stiffness index (B) before and after 12 weeks dietary intervention. Data are expressed as means \pm SE.

Figure 2. Aortic PWV before and after a 12-week dietary intervention. Data are expressed as means \pm SE.

Figure 3. Plasma endothelin-1 concentrations before and after a 12-week dietary intervention. Data are expressed as means \pm SE.

Figure 4. Plasma nitrite/nitrate (NOx; the stable end product of nitric oxide) concentrations before and after a 12-week dietary intervention. Data are expressed as means $\pm \mathrm{SE}$. 
Figure 1
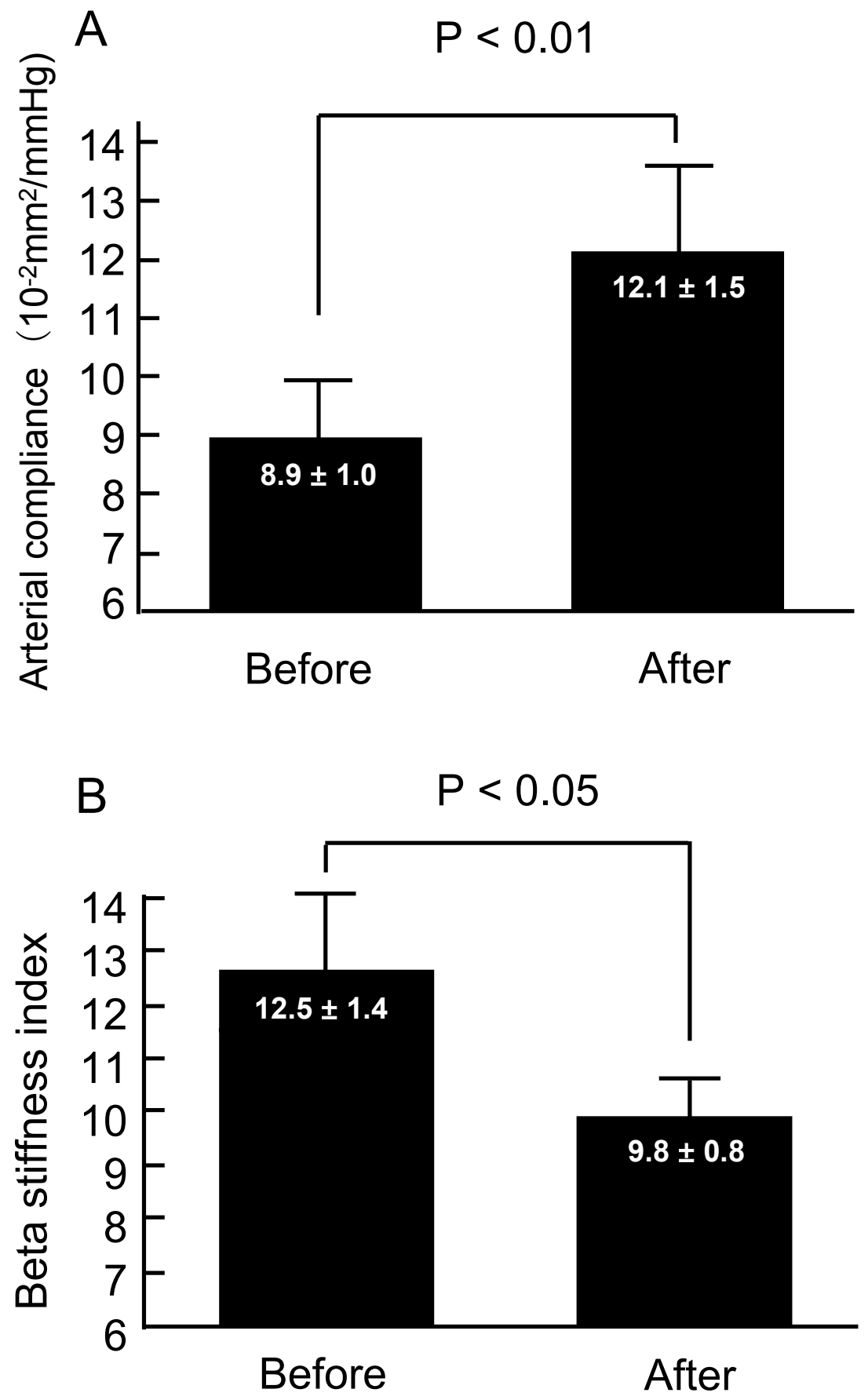
Figure 2

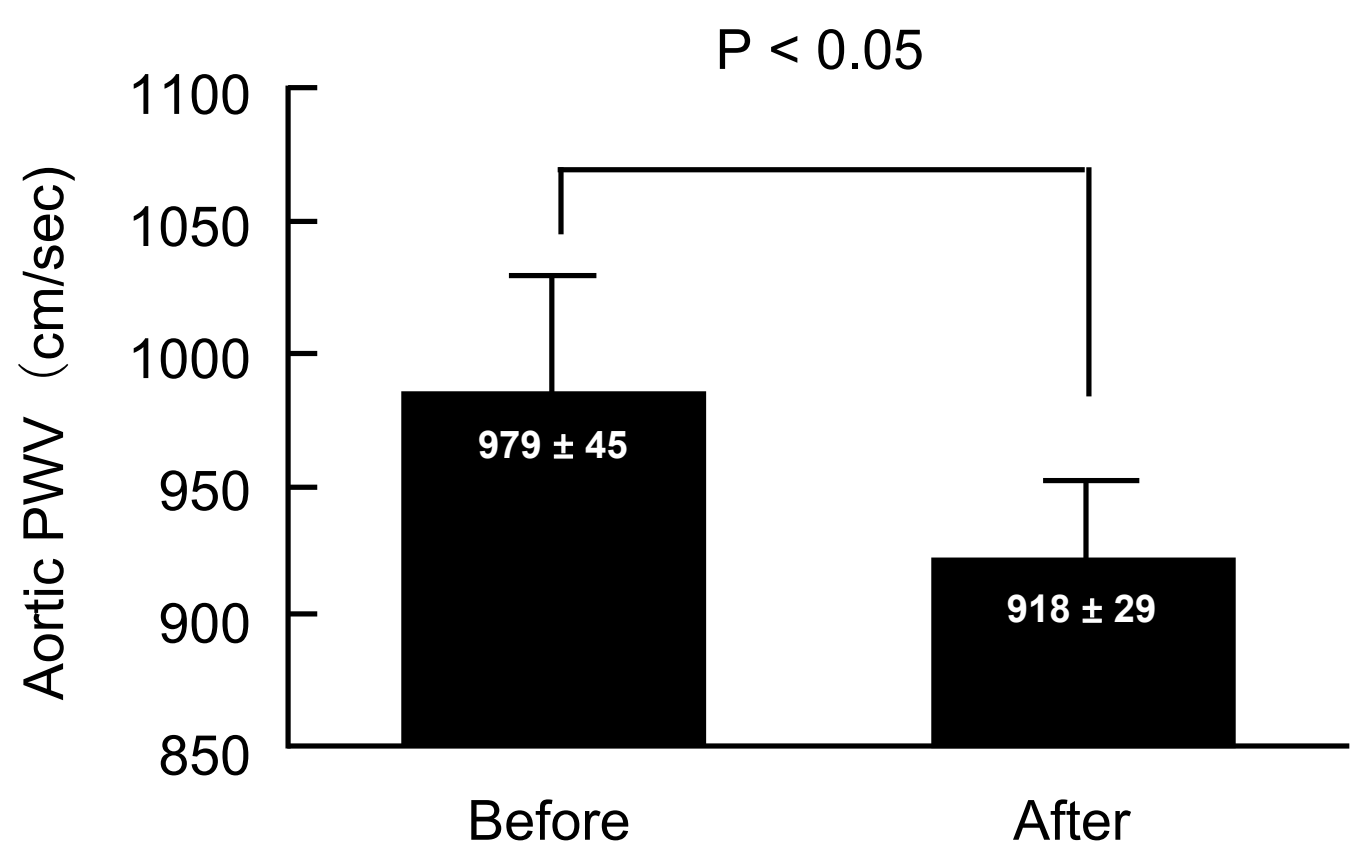


Figure 3

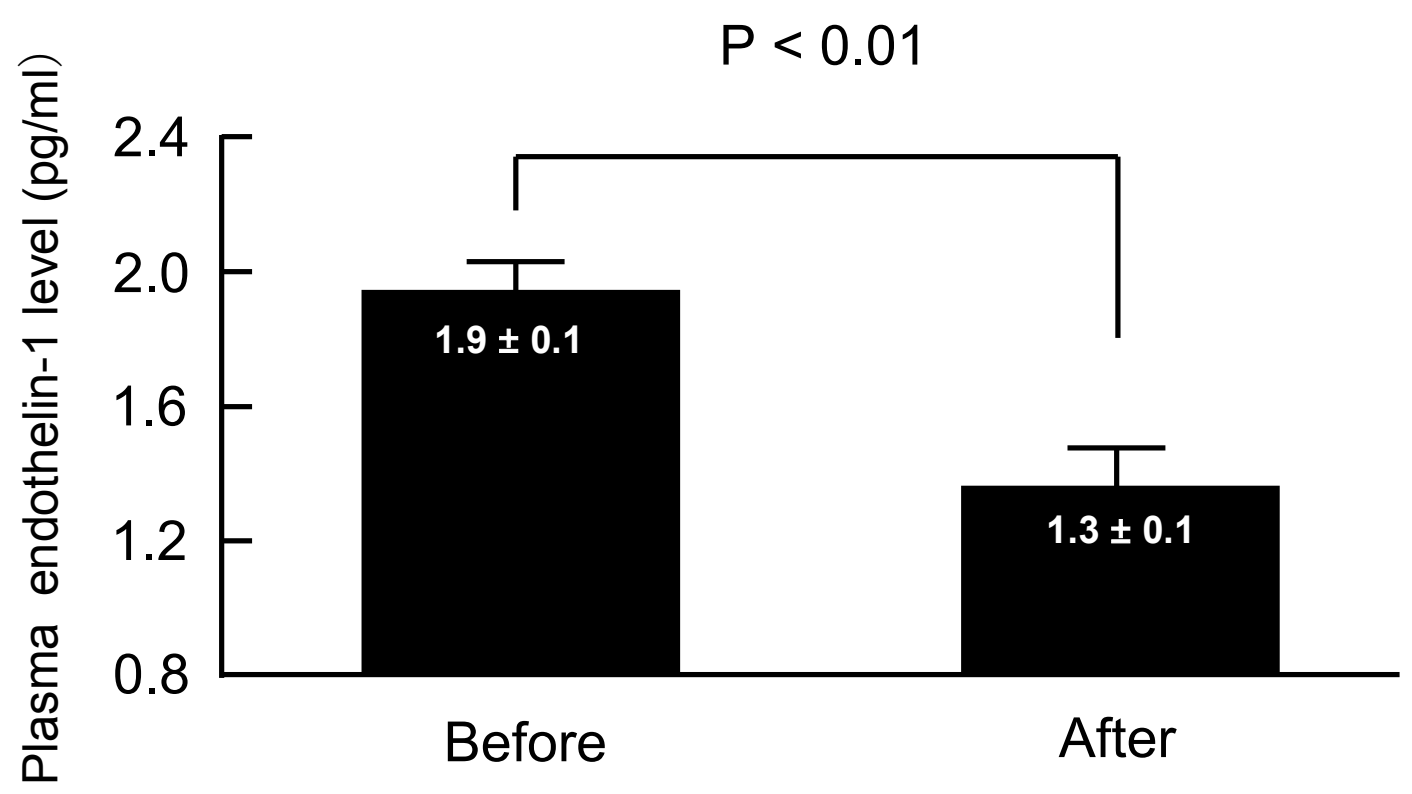


Figure 4

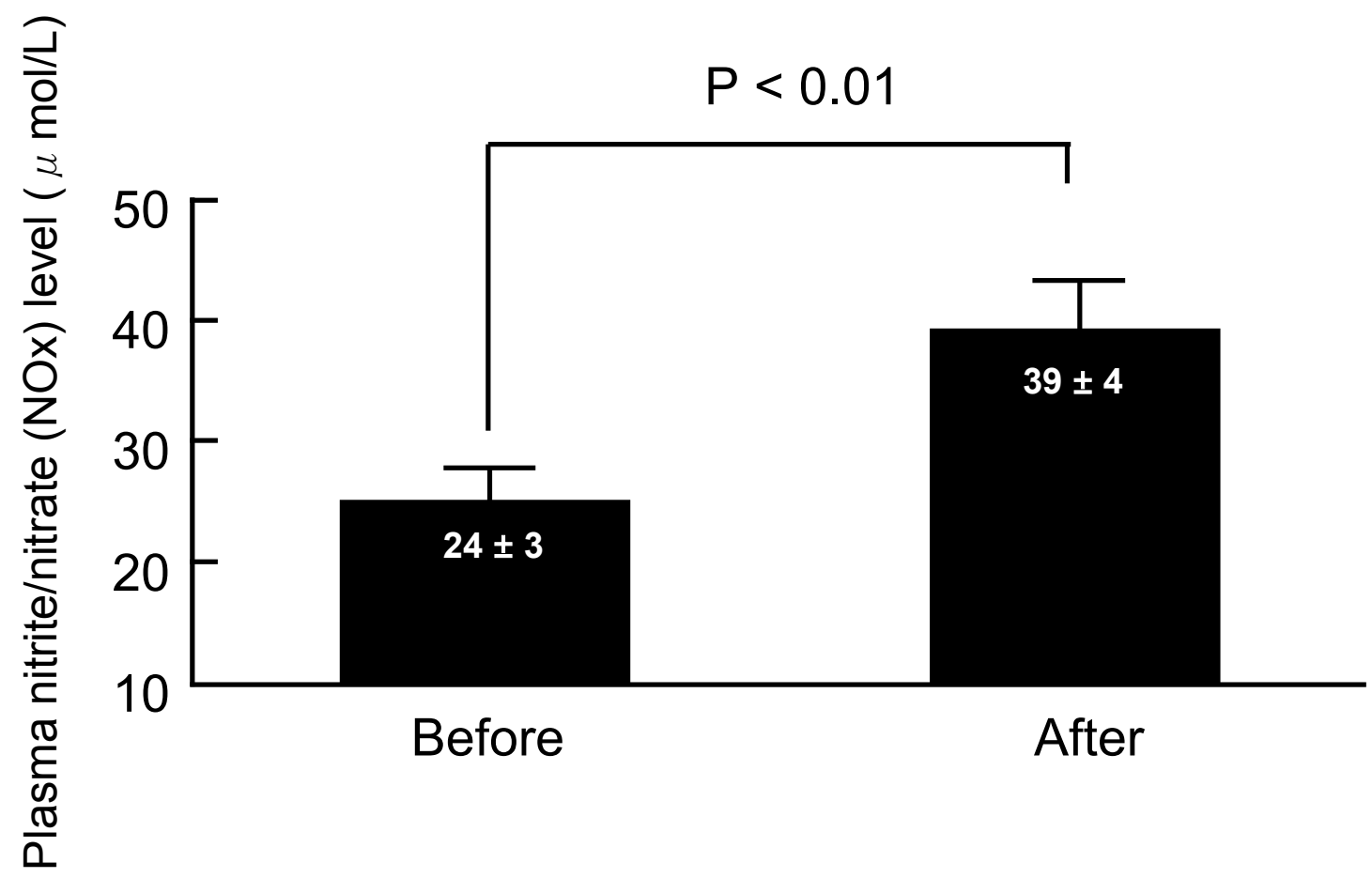


Table 1. Physiological characteristics of obese men before and after the weight reduction program

\begin{tabular}{|c|c|c|c|c|c|c|}
\hline \multirow[b]{2}{*}{ Age, years } & \multicolumn{3}{|c|}{ Before } & \multicolumn{3}{|c|}{ After } \\
\hline & 45 & \pm & 2 & & - & \\
\hline Height, cm & 172 & \pm & 2 & & - & \\
\hline Weight, kg & 88 & \pm & 3 & 80 & \pm & $4^{\star *}$ \\
\hline Body mass index, $\mathrm{kg} / \mathrm{cm}^{2}$ & 30 & \pm & 1 & 27 & \pm & $1^{\star \star}$ \\
\hline Visceral fat area, $\mathrm{cm}^{2}$ & 169 & \pm & 9 & 119 & \pm & $10^{* *}$ \\
\hline Subcutaneous fat area, $\mathrm{cm}^{2}$ & 256 & \pm & 21 & 196 & \pm & $25^{\star \star}$ \\
\hline Total fat area, $\mathrm{cm}^{2}$ & 424 & \pm & 25 & 315 & \pm & $32^{* *}$ \\
\hline Total cholesterol, mg/dl & 217 & \pm & 12 & 196 & \pm & $13^{\star}$ \\
\hline HDL-cholesterol, mg/dl & 56 & \pm & 3 & 57 & \pm & 4 \\
\hline LDL-cholesterol, mg/dl & 130 & \pm & 11 & 121 & \pm & 12 \\
\hline Triglyceride, mg/dl & 157 & \pm & 24 & 92 & \pm & $11^{\star *}$ \\
\hline Blood glucose, mg/dl & 100 & \pm & 14 & 93 & \pm & $7^{\star}$ \\
\hline
\end{tabular}

Data are expressed as means \pm SE. Significant difference vs. before dietary intervention, ${ }^{\star} P<0.05 ;{ }^{*} P<0.01$. 
Table 2. Hemodynamics of obese men before and after weight reduction program

\begin{tabular}{lrrrrrr}
\hline & \multicolumn{3}{c}{ Before } & \multicolumn{3}{c}{ After } \\
\hline Systolic blood pressure, $\mathrm{mmHg}$ & 137 & \pm & 4 & 123 & \pm & $6^{* *}$ \\
Diastolic blood pressure, $\mathrm{mmHg}$ & 80 & \pm & 3 & 72 & \pm & $3^{* \star}$ \\
Mean blood pressure, $\mathrm{mmHg}$ & 99 & \pm & 3 & 89 & \pm & $4^{* \star}$ \\
Pulse pressure, $\mathrm{mmHg}$ & 57 & \pm & 4 & 50 & \pm & $4^{* \star}$ \\
Heart rate, bpm & 79 & \pm & 5 & 63 & \pm & $3^{* *}$ \\
\hline
\end{tabular}

Data are expressed as means \pm SE. Significant difference vs. before dietary intervention, ${ }^{\star \star} P<0.01$. 